\title{
T2 formula in a highly myopic population, comparison with other methods and description of an improved approach for estimating corneal height
}

\author{
Carlos Alberto Idrobo-Robalino ${ }^{1 *}$ D, Gisella Santaella ${ }^{2}$ and Ángela María Gutiérrez ${ }^{2}$
}

\begin{abstract}
Background: To determine the accuracy of the T2 formula as applied to highly myopic eyes, to compare the T2 formula to the SRK $/ T$ and Holladay 1 formulas, and to describe possible ways to improve the estimation of corneal height and prediction error in two settings, the Hadassah Hospital, Ophthalmology Department, Jerusalem, Israel and Clínica Barraquer, Bogotá, Colombia.

Methods: In this retrospective case series, optical biometer measurements were taken for 63 highly myopic patients (> $25 \mathrm{~mm}$ ) undergoing uneventful crystalline lens phacoemulsification and insertion of an acrylic intraocular lens. Prediction errors were obtained, with estimations of $\pm 0.50 \mathrm{D}, \pm 1.00 \mathrm{D}$, and greater than \pm $2.00 \mathrm{D}$. A method to improve the corneal height calculation is described.

Results: The SRK $T$ formula (mean absolute error $[\mathrm{MAE}]=0.418$; median absolute error $[\mathrm{MedAE}]=0.352$ ) was the most accurate, followed by the T2 (MAE $=0.435$; MedAE $=0.381)$ and Holladay $1(\mathrm{MAE}=0.455$; MedAE $=0.389)$ formulas. Both the SRK/T and T2 formulas overestimated corneal height, but values were higher with the T2 formula. Corneal height was more precisely estimated using an alternative method that, when combined with axial length optimization, resulted in lower MAE (0.425) and MedAE (0.365) values than when applying the T2 formula alone.
\end{abstract}

Conclusions: The T2 formula seems to be less accurate than the SRK $T$ formula in highly myopic eyes. An improved corneal height estimation method is described for the the T2 formula.

Keywords: T2 formula, High myopia, Corneal height estimation, Cataract surgery, Intraocular lens calculation

\section{Background}

Highly myopic eyes have a long axial length (L); (> 25 $\mathrm{mm}$ ), a deep anterior chamber depth (ACD), and a floppy capsular bag, therefore, calculating the intraocular lens (IOL) power of these eyes is challenging and often results in a postoperative hyperopic surprise. The use of partial coherence interferometry [1] together with specific formulas (e.g. Barrett Universal II [2] and

\footnotetext{
* Correspondence: caidrobo22@gmail.com

Presented as a Lecture at the Meeting of Clínica Barraquer in the XXXVII, Congreso Nacional e Internacional de Oftalmología, Sociedad Colombiana de Oftalmología, Cartagena de Indias, Colombia, August 2016.

${ }^{1}$ Hospital Eugenio Espejo, Avenida Gran Colombia 170403, Quito, Ecuador

Full list of author information is available at the end of the article
}

Haigis [3]) are strategies to improve the IOL estimation in these cases.

The SRK/T formula is a well-known method with evidence reporting its accuracy in cases of high myopia [2]. The size of the postoperative anterior chamber and the position of the IOL are predicted by the SRK/T using the following concepts: 1) The corneal height $(\mathrm{H})$, is a model in which the cornea is regarded as a section of a sphere, the base of which forms a plane at the level of the anterior iris, therefore $\mathrm{H}$ can be defined as the distance from the anterior surface of the iris to the central cornea, in the SRK/T paper measures dealing with this value included the corneal thickness [4]. 2) Corrected Axial Length (LCOR): The SRK/T assumes that the vitreous chamber size undergoes a 
greater elongation than the anterior segment, As a result, this formula applies a correction factor in eyes longer than $24.2 \mathrm{~mm}$ of axial length which allows for a more accurate estimation of ACD in the long eye, this adjustment is used as part of the corneal height $(\mathrm{H})$ estimation [4]. 3) Offset: Below the iris, and with the IOL in position, the offset is the distance from the iris plane to the optical plane of the IOL.

In spite of the advantages of the SRK/T, authors like Haigis [5] observed that it was not as effective in certain situations. For instance, in the calculation of the $\mathrm{ACD}$, when the corneal width is two times greater than the corneal radius, the formula attempts to calculate the square root of a negative number, a phenomenon termed "imaginary ACD." This event is controlled by changing the described negative value to zero, an adjustment that only represents a partial solution, and that induces a non-physiological behavior, called the "SRK/T cusp." [6]

The T2 formula was developed as a method which would tackle the pitfalls of the SRK/T, its authors describe two sources of error for the original formula [1]: LCOR reversal, where LCOR progressively decreases as AL values exceed $36.2 \mathrm{~mm}$; and [2] the SRK/T cusp, corrected by replacing steps 2 to 4 in the original SRK/T formula with a regression formula for corneal height [6] (from now on called H2). The T2 formula corrects estimation errors of $\mathrm{H}$ but its benefits are not as evident as expected in long eyes $[7,8]$.

An important feature of the design of the $\mathrm{T} 2$ equation is that it uses $\mathrm{L}$ without any correction (avoiding the LCOR step from the SRK/T), and the keratometry. Interestingly, a second formula for the corneal height was developed in the original report on the T2 formula, which does include LCOR (termed H2.2 herein) and which will be of special interest in this paper. Appendix 2 presents all aforementioned equations.

The Holladay 1 formula has also been successfully used in normal and myopic eyes [4], and it has been included in the present study for comparison purposes, due to its similar design to the SRK/T.

The present investigation compared the outcomes of the SRK/T, T2, and Holladay 1 formulas in highly myopic eyes. In addition, it analyzed the SRK/T and
T2 formulas in order to find options to improve the prediction of $\mathrm{H}$ in very long eyes.

\section{Methods}

An observational retrospective chart review was performed. This review included 63 highly myopic patients $(>25.00 \mathrm{~mm})$, who underwent uneventful crystalline lens phacoemulsification and IOL insertion at one of two clinics: the Hadassah Ein Keren Ophthalmology Clinic, Jerusalem, Israel (39 cases from June 2012 to January 2014) and the Clínica Barraquer, Bogotá, Colombia (24 cases from February 2013 to November 2015). Institutional review board approval was obtained, and all methods adhered to the Helsinki Declaration. Inclusion criteria were as follows: highly myopic eyes $(\mathrm{L}>25 \mathrm{~mm})$, Alcon Acrisoft ${ }^{\circ}$ SN60WF acrylic IOL in-the-bag implants, and postoperative visual acuity $\geq 20 / 40$. Exclusion criteria were as follows: absent or inadequate optic biometry and/or conditions affecting best corrected visual acuity (e.g. choroidal neovascularization, optic atrophy, etc.). Myopic retinal degeneration and glaucoma were reasons for exclusion only if severely impairing.

The measured variables were as follows: $\mathrm{L}$ and keratometry (measured with Carl Zeiss IOL Master ${ }^{\circ}$ Optical Biometer); preoperative and postoperative best corrected visual acuity (measured with ETDRS chart and converted to LogMAR notation using an online tool [http://www.myvisiontest.com/logmar.php]; postoperative refraction (measured at minimum one month postoperation). The Holladay 1, SRK/T, and T2 formulas were included for assessments. The applied A-constant and Surgeon Factor were respectively 119.0 and 1.84 (based on recommendations from the User Group for Laser Interference Biometry) [9].

The IOL powers for predicted refraction and emmetropia were estimated. Prediction error was defined as the difference between the refractive error calculated by the formula and the stable postoperative refraction. Calculations were performed using verified formulas developed by Dr. Richard Sheard (Microsoft Excel Functions Add-In Version 4.2).

The estimation of errors was as follows: Mean Error $(\mathrm{ME})$ was made equal to zero by changing the lens factor

Table 1 Demographics of the two studied groups

\begin{tabular}{|c|c|c|c|c|c|}
\hline Group & Ethnicity & Mean Age & Gender & Laterality & N \\
\hline Hadassah Ein Keren Hospital, Jerusalem, Israel & Jewish; Arabic & $\begin{array}{l}68.67 \text { yo, SD } \pm 10.25 \\
\text { Min: } 43 \\
\text { Max: } 85\end{array}$ & $\begin{array}{l}\text { Male: } 43.85 \% \\
\text { Female: } 56.41 \%\end{array}$ & $\begin{array}{l}\text { Right: } 58.97 \% \\
\text { Left: } 41.02 \%\end{array}$ & 39 \\
\hline Clínica Barraquer, Bogotá-Colombia & Latin American - Hispanic & $\begin{array}{l}60.41 \text { yo, SD } \pm 12.14 \\
\text { Min: } 37 \\
\text { Max : } 81\end{array}$ & $\begin{array}{l}\text { Male: } 41.66 \% \\
\text { Female: } 58.33 \%\end{array}$ & $\begin{array}{l}\text { Right: } 66.6 \% \\
\text { Left: } 33.3 \%\end{array}$ & 24 \\
\hline
\end{tabular}

yo years old, SD Standard Deviation, Min Minimum, Max Maximum, $n$ Number of eyes Studies 
Table 2 Variables included in the present study

\begin{tabular}{lllll}
\hline Variable & Mean & Standard Deviation & Minimum & Maximum \\
\hline PreOp VA (Logmar) & 0.494 & 0.346 & 0.041 & 0 \\
PostOp VA (Logmar) & 0.101 & 0.1043 & 0 & 0.301 \\
Flat K & $42.99 \mathrm{D}$ & $1.61906832 \mathrm{D}$ & $39.38 \mathrm{D}$ & $46.81 \mathrm{D}$ \\
Steep K & $44.09 \mathrm{D}$ & $1.76891495 \mathrm{D}$ & $40.23 \mathrm{D}$ & $48.5 \mathrm{D}$ \\
Mean K & $43.54 \mathrm{D}$ & $1.62941283 \mathrm{D}$ & $40.08 \mathrm{D}$ & $47.2 \mathrm{D}$ \\
L & $26.94 \mathrm{~mm}$ & $1.107 \mathrm{~mm}$ & $25.22 \mathrm{~mm}$ & $30.08 \mathrm{~mm}$ \\
\hline
\end{tabular}

PreOp Preoperative, PostOp Postoperative, VA Visual acuity, K Keratometry, $L$ Axial length; $n=63$

individually for each formula, this was achieved using the Excel software's Data/What If Analysis/Goal Seek function [10], after this procedure, constants obtained were: A constant for SRK/T: 119.21; A constant for T2: 119.23; A Constant for T2 formula including $\mathrm{H} 2.2$ and Wang's AL optimization (described below): 118.63; Surgeon Factor for Holladay 1: 2.27.

After the mean errors were zeroed out, all negative values were converted to positive and the mean absolute error (MAE) was reported for each formula. Then, Median Absolute Error (MedAE) was calculated. Standard, minimum and maximum errors were estimated, together with the percentage of eyes with prediction errors $\leq \pm$ 0.50 diopter $(\mathrm{D}), \leq \pm 1.00 \mathrm{D}$, and $\leq \pm 2.00 \mathrm{D}[10]$.

The overall sample was analyzed to avoid subgroup bias. $\mathrm{H}$ was calculated using steps 2 to 4 of the SRK/T formula [4] (termed hereafter as HSRK/T), and two equations described by Sheard et al. [6] ( $\mathrm{H} 2$ and $\mathrm{H} 2.2$ ). Correlative analyses were performed using commercially available software (Excel 2013, SPSS v.17.0).

Eyes with previous corneal surgery or corneal diseases, and preoperative pathologic changes affecting central vision were excluded. Foveal and perifoveal integrity together with confirmation of stability of any condition were required before inclusion in the sample for analysis.

\section{Results}

\section{Sample description}

The demographics of each sample group (i.e. 39 cases from Hadassah Ein Keren Hospital and 24 from Barraquer Clinic) are detailed in Table 1.

The pre and post-operative statuses of the assessed variables are summarized in Table 2.
The target preoperative refraction had a mean of 1.171 (Min - 5 Max: 0.68, SD 1.330). Whereas the postoperative refraction had a mean Sphere of -0.783 (Min - 4.25; Max:1.5; SD 1.382) and a mean Cylinder of 0.900 (Min - 4 Max: 0, SD 0.745).

Preoperative pathology was found in eight out of 63 eyes $(12.69 \%)$ : one case of uveitis $(1.59 \%)$, one case of temporary diplopia $(1.59 \%)$, one case of pseudo exfoliation syndrome (1.59\%), one case with peripheral lesions requiring laser treatment (1.59\%), and one case of extrafoveal choroidal neovascularization (1.59\%). Three patients presented with atrophic macular changes outside the fovea (4.76\%). Any pathology found was confirmed to be stable and not affecting visual acuity before cataract surgery took place, these cases were allowed in the analysis group provided that none of the changes was found to affect visual acuity.

\section{Ranking of formulas}

Of the tested equations, the most accurate was the SRK/ $\mathrm{T}$ formula $($ MedAE $=0.352)$, followed by $\mathrm{T} 2($ MedAE $=$ $0.381)$ and Holladay 1 (MedAE $=0.389)$ formulas (Table 3, Fig. 1). Lin's correlation [11] factor was used to analyze the MedAE of the three methods (Table 4).

A substantial correlation was found between the T2 and SRK/T formulas. Correlations between the SRK/T and Holladay 1 formulas and between the Holladay 1 and T2 formulas were also substantial, but with only moderate lower limits of the confidence intervals.

\section{Analysis of calculation methods}

Since the main difference between the T2 and SRK/T formulas is the estimation of $\mathrm{H}$, the behaviors of $\mathrm{L}$ and keratometry were analyzed respect to Corneal Height.

Table 3 Summary of the prediction error in the present study

\begin{tabular}{|c|c|c|c|c|c|c|c|c|c|}
\hline Formula & MAE & Standard Deviation & Minimum & Maximum & MedAE & $\leq \pm 0.50 \mathrm{D}$ & $\leq \pm 1.0 \mathrm{D}$ & $\begin{array}{l}\text { Sum of errors } \\
\leq \pm 0.50 \mathrm{D}+\leq \pm 1.0 \mathrm{D}\end{array}$ & $>2.00 \mathrm{D}$ \\
\hline SRK厂 & 0.418 & 0.327 & 0.003 & 1.359 & 0.352 & $71.42 \%$ & $20.63 \%$ & $92.05 \%$ & $7.93 \%$ \\
\hline Holladay1 & 0.455 & 0.314 & 0.037 & 1.404 & 0.389 & $61.90 \%$ & $31.74 \%$ & $93.64 \%$ & $6.35 \%$ \\
\hline $\mathrm{T} 2$ & 0.435 & 0.328 & 0.014 & 1.389 & 0.381 & $69.84 \%$ & $22.22 \%$ & $92.06 \%$ & $7.94 \%$ \\
\hline
\end{tabular}

MAE Mean absolute error, MedAE Median absolute error, T2 T2 formula, $n=63$ 


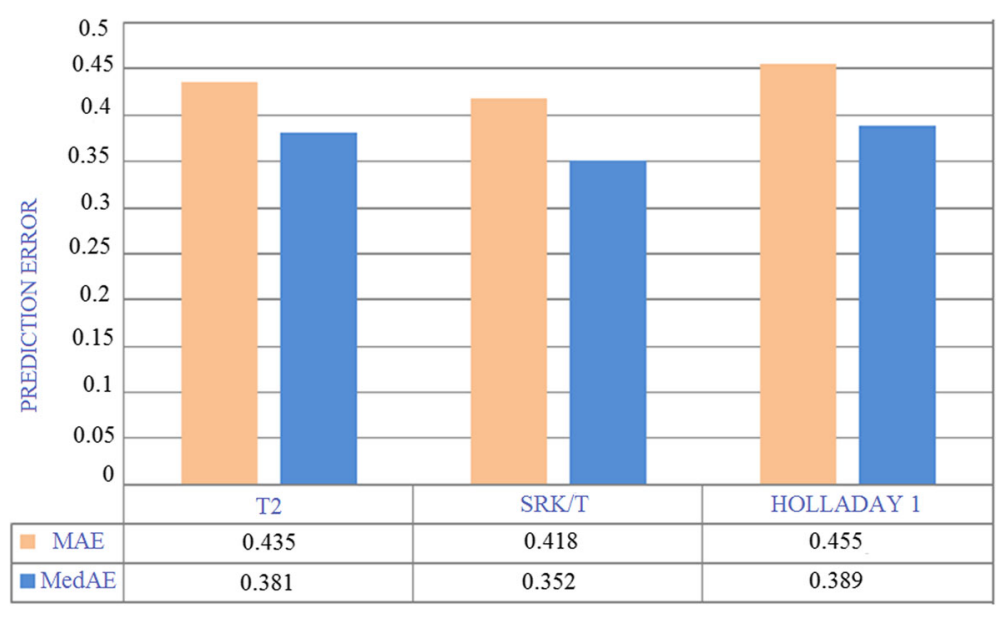

FORMULA

Fig 1 Median and Mean Absolute Error of the T2, SRK/T and Holladay 1 formulas Abbreviations: MAE: Mean Absolute Error; MedAE: Median Absolute Error; T2: T2 formula; SRK/T: SRK T formula, $n=63$

$\mathrm{L}$ is used without any modification in $\mathrm{H} 2$, while an adjusted L (LCOR) is required by the HSRK/T formula. A correlative analysis was performed between both $\mathrm{H}$-calculation methods and $\mathrm{L}$, with the results being a very low correlation between HSRK/T and L (Table 5) but a strong positive correlation between $\mathrm{H} 2$ and $\mathrm{L}(r=0.808 ; p<0.05)$.

This finding is important for the following reasons [1]: it suggests that $\mathrm{L}$ has a strong effect on the estimation of $\mathrm{H}$ calculated with the method included in the T2 formula [2]; it might explain the higher MedAE seen in highly myopic eyes with the T2 formula; and [3] it indicates that LCOR may be why L has less impact when $\mathrm{H}$ is estimated with the SRK/T approach.

In summary, modifying the calculation of $\mathrm{H}$ in the $\mathrm{T} 2$ formula improves its accuracy, resulting in a lower MedAE in eyes with normal L. However, the benefit of this adjustment seems to be lost in longer eyes, probably due to the effect of $\mathrm{L}$ on the estimation of H. On the other hand, the SRK/T formula seems to be less affected by an extreme $\mathrm{L}$, which could be associated with the inclusion of LCOR in its design.

The second variable needed to calculate $\mathrm{H}$ is the keratometry. The average keratometry was found to have a strong positive relationship with HSRK/T $(r=0.805, p<0.05)$, but a negligible correlation with $\mathrm{H} 2(r=0.265, p<0.05)$.

Table 4 Lin's correlation coefficient of the median absolute error of the methods used in the present study

\begin{tabular}{lll}
\hline & T2 & HOLLADAY 1 \\
\hline SRK $/ \mathrm{N}$ & $\rho_{c}=0.9829$ & $\rho_{c}=0.9537$ \\
& $95 \% \mathrm{Cl}=0.9720$ to 0.9896 & $95 \% \mathrm{Cl}=0.9253$ to 0.9715 \\
T2 & & $\rho_{c}=0.9575$ \\
& & $95 \% \mathrm{Cl}=0.9311$ to 0.9739
\end{tabular}

$\rho_{c}:$ Lin's concordance correlation coefficient, $95 \%$ Cl: $95 \%$ confidence interval. $n=63$

\section{Improvement options \\ Corneal height $(H)$}

The performed analyses suggested that the presence of LCOR reduces the impact of extreme AL values in the estimation of $\mathrm{H}$. Therefore, including the corrected $\mathrm{AL}$ in the $\mathrm{T} 2$ formula might improve its behavior in long eyes. Therefore, a formula which might both, solve the SRK/T cusp problem and include LCOR was needed. The easiest way to complete this task was using the second regression formula described by Sheard et al. in the original paper on the $\mathrm{T} 2$ formula. This second regression formula was excluded from the final T2 method because of its slightly lower correlation [6]. In the present study, this formula is named $\mathrm{H} 2.2$ and is calculated as follows:

$\mathrm{H} 2.2=-11.980+0.38626 \times \mathrm{LCOR}+0.14177 \times \mathrm{K}$

Estimations of $\mathrm{H}$ using the $\mathrm{H} 2.2$ formulas were compared with results obtained using the HSRK/T and $\mathrm{H} 2$ formulas (Fig. 2, Table 6). The H2.2 method reduced the mean $\mathrm{H}$ value and the reported range of values.

Table 5 Correlation between different methods of Corneal Height estimation and associated variables

\begin{tabular}{lll}
\hline & Axial Length & Mean Keratometry \\
\hline HSRKT & $r=0.224$ & $r=0.805$ \\
& $p=0.078$ & $p<0.01$ \\
$\mathrm{H} 2$ & $r=0.808$ & $r=0.265$ \\
& $p<0.01$ & $p<0.05$ \\
$\mathrm{H} 2.2$ & $r=0.425$ & $r=0.695$ \\
& $p<0.01$ & $p<0.01$ \\
\hline
\end{tabular}

HSRK/T: Corneal height estimation using SRK/T, H2: Corneal height estimation using T2, H2.2: Corneal height estimation using the alternative T2 formula 


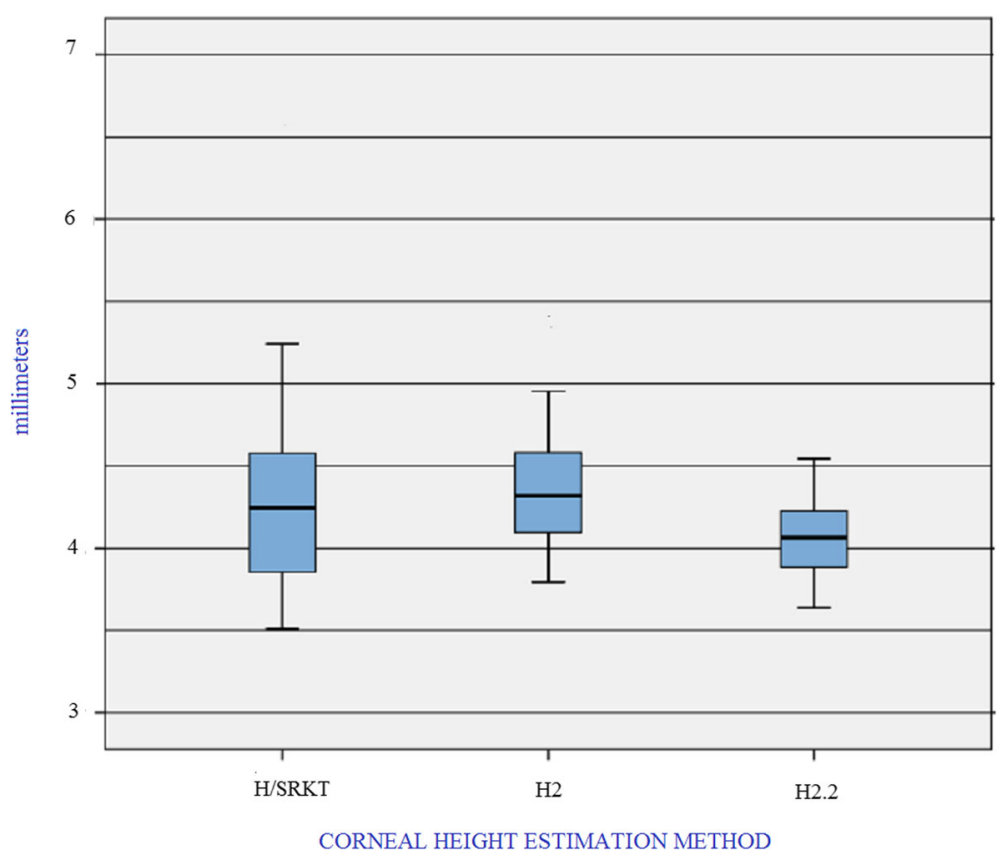

Fig 2 Box plot of Corneal Height estimations using SRKT, T2 and the alternative Corneal Height method described. Abbreviations: HSRK/T: Corneal Height estimation using steps 2 to 4 of the SRK T formula; H2: Corneal height estimation using equation number 1 for $\mathrm{H}$ described by Sheard et al. [6] and programed in the T2 formula. H2.2: Corneal height estimation using equation number 2 for $\mathrm{H}$ described by Sheard et al. [6] and applied in the present work. $n=63$

Statistically significant differences were found between the $\mathrm{H} 2.2$ and $\mathrm{H} 2$ formulas $(p<0.005)$, as well as between the H2.2 and HSRK/T formulas $(p<0.005)$. A moderate correlation was found between $\mathrm{H} 2.2$ and average keratometry $(r=0.695, p<0.05)$, and a low correlation was found between $\mathrm{L}$ and $\mathrm{H} 2.2(r=0.425, p<0.05)$.

These results suggest that the H2.2 formula might improve $\mathrm{H}$ estimations, reducing the mean $\mathrm{H}$, the range of extreme values, and the influence of very high keratometry and $\mathrm{L}$ values.

When H2.2 was used to estimate IOL, the MAE and MedAE were respectively 0.433 and 0.3815 (Table 7).

While these results are only slightly better than T2 formula, a better estimation of $\mathrm{H}$ in highly myopic patients is obtained.

\section{Optimized axial length}

An additional approach to improve results of the T2 formula in highly myopic eyes is to optimize axial length.

Table 6 Corneal Height estimation using three methods

\begin{tabular}{lllll}
\hline & Minimum & Maximum & Mean & Standard Deviation \\
\hline HSRKT & 3.5101 & 6.6086 & 4.2713 & \pm 0.5490 \\
H2 & 3.7947 & 5.4057 & 4.3567 & \pm 0.3503 \\
H2.2 & 3.6395 & 4.7624 & 4.0631 & \pm 0.23624 \\
\hline
\end{tabular}

HSRK/T: Corneal height estimation using SRK/T, H2: Corneal height estimation using T2, H2.2: Corneal height estimation using the alternative T2 formula, $n=63$
Since H2.2 includes LCOR, the method described by Wang L et al. [12] for the SRK/T formula can be used directly. When this approach was tested, the MedAE and MAE were even lower than obtained with H2.2 alone (Table 7).

\section{Discussion}

The accuracy of the SRK/T formula in highly myopic patients has long been established $[2,7,13]$, in spite of this, flaws estimating $\mathrm{H}$ have been described $[5,6]$. The T2 formula, developed by Sheard et al. [6], improves $\mathrm{H}$ prediction and significantly reduces the prediction error in normal eyes. It could, therefore, be assumed that the T2 formula would perform better than the SRK/T formula among highly myopic patients, but the present investigation found that SRK/T formula could still be a better choice.

The SRK/T approach for estimating $\mathrm{H}$ utilizes keratometry and L, The axial length estimation is corrected using LCOR when it is higher than $24.2 \mathrm{~mm}$ [4]. The resulting $\mathrm{H}$ value in highly myopic patients includes errors such as the H cusp and LCOR reversal [6], both of which result in a far greater $\mathrm{H}$ estimation than what could be considered normal, even for myopic patients. This is evident when studies of corneal height measurement in vivo are considered. For instance, Dong Hyun Kim et al. [14] reported a mean $H$ value of $3.71 \pm 0.23$ $\mathrm{mm}$, measured by optical coherence tomography, in 
Table 7 Prediction error applying T2 with the alternative corneal height estimation method and optimization of axial length

\begin{tabular}{|c|c|c|c|c|c|c|c|c|c|}
\hline Formula & MAE & Standard Deviation & Minimum & Maximum & MedAE & $\leq \pm 0.50 \mathrm{D}$ & $\leq \pm 1.0 \mathrm{D}$ & $\begin{array}{l}\text { Sum of errors } \\
\leq \pm 0.50 D+\leq \pm 1.0 \mathrm{D}\end{array}$ & $>2.00 \mathrm{D}$ \\
\hline T2 using $\mathrm{H} 2.2$ alone & 0.433 & $\pm 0,0117$ & 0.0032 & 1.3856 & 0.3816 & $69.84 \%$ & $22.22 \%$ & 92.06 & $7.93 \%$ \\
\hline T2 using H2.2 and optimized L & 0.425 & \pm 0.3318 & 0.0025 & 1.382 & 0.3648 & $68.25 \%$ & $23.81 \%$ & 92.06 & $0 \%$ \\
\hline
\end{tabular}

$\mathrm{H} 2.2$ = Corneal height estimation according to the alternative T2 formula, Optimized L: Adjustment of $\mathrm{L}$ according to Wang $\mathrm{L}$ et al. [12] $n=63$

patients with a mean L of $28.00 \mathrm{~mm}$. Another study comparing the eyes of anisometric patients reported that ACD did not differ greatly between the shorter and longer eye, even when very highly myopic patients were included. Therefore, ACD and $\mathrm{H}$ values in highly myopic patients do not differ extremely from the values for normal eyes. The increased L in highly myopic eyes depends mostly on the vitreous cavity and not on an extremely deep anterior chamber [15].

The T2 formula solves the $\mathrm{H}$ cusp problem [6], but the equation used in the original report did not include LCOR. According to the findings of the present study, LCOR might be an important factor related to the higher precision of the SRK/T formula in highly myopic eyes. In addition, the $\mathrm{H} 2$ equation, included in the $\mathrm{T} 2$ formula, resulted in a higher mean $\mathrm{H}$ than the method used by the SRK/T formula. This could partially explain the higher MedAE and MAE values when applying the T2 formula to highly myopic eyes.

In this regard, the solution to improve the $\mathrm{T} 2$ prediction error proposed in the present study includes two parts. First, since LCOR helps improve the $\mathrm{H}$ estimate in the SRK/T formula, this step was included in the T2 estimation of $\mathrm{H}$, specifically using the second regression formula described in the report on the T2 formula [6]. The result of this change was a more precise $\mathrm{H}$ estimation than that obtained using either HSRK/T or the regular $\mathrm{H} 2$ method. The second step was to improve $\mathrm{L}$ estimation. This goal was accomplished by using a published L optimization equation for SRK/T [6], which resulted in lower MAE and MedAE values than those observed using $\mathrm{T} 2$ alone.
An issue of including LCOR in the T2 formula might be that in very long eyes (i.e. $\mathrm{L}>36.2 \mathrm{~mm}$ ) the LCOR reversal phenomenon appears, therefore a formula that uses the SRK/T platform together with additional solutions should assess this concern to best fit the requirements of long eyes. Methods to optimize L could be applied directly to the T2 formula or the described H2.2 method.

Other studies have tested the T2 formula in different settings (Table 8), and no definitive consensus exists regarding the accuracy of the T2 vs SRK/T formulas in long eyes. One study found better results using SRK/T [7], while another described better accuracy using T2 [8]. The results of the present study are similar to previous analyses of the SRK/T and Holladay 1 formulas $[2,7]$, but new information is provided in relation to calculating H. Suggestions for improving IOL calculations in highly myopic patients are provided. Despite these contributions, an important limitation of the present study is the relatively small sample size. This limitation is due to the relative infrequency of highly myopic eyes, even among very large sample pools. The inclusion of more highly myopic cases may be needed to clarify the presented observations and to develop necessary optimization formulas.

Calculating the IOL in highly myopic eyes is still a complicated issue, and even with modern formulas, errors still exist. This reality underscores the importance of continued investigation and improvement in this subject. The SRK/T formula is one of the most accurate for long-eyed patients with the advantage of being readily available in different settings. Therefore, improving this

Table 8 Comparison of studies that include the T2 formula

\begin{tabular}{|c|c|c|c|c|}
\hline & Kane, et al. (2016) & Cooke \& Cooke (2016) & Sheard et al. (2010) & Present study \\
\hline $\begin{array}{l}\text { Total studied eyes } \\
\text { (\# of long-eyes) }\end{array}$ & $3241(77)$ & $1454(54)$ & 11189 (not target of study) & $63(63)$ \\
\hline Long eye definition & $>26.0 \mathrm{~mm}$ & $\begin{array}{l}\text { PCI (25.97-29.44 mm) } \\
\text { OCLR (26.02-29.51 mm) }\end{array}$ & Not target of study & $>25.0 \mathrm{~mm}$ \\
\hline Formulas: MAE/MedAE & $\begin{array}{l}\text { T2: } 0.498 / 0.440 \\
\text { SRK/T: } 0.484 / 0.419 \\
\text { Holladay 1: } 0.586 / 0.441\end{array}$ & $\begin{array}{l}\text { PCl group } \\
\text { T2: } 0.319 / 0.269 \\
\text { SRK/T: 0.399/0.368 } \\
\text { Holladay 1: } 0.495 / 0.473 \\
\text { OLCR group } \\
\text { T2: } 0.293 / 0.251 \\
\text { SRK/T: } 0.392 / 0.344 \\
\text { Holladay } 1: 0.505 / 0.479\end{array}$ & $\begin{array}{l}\text { T2: MAE }=0.306 \\
\text { SRK T: } M A E=0.3229\end{array}$ & $\begin{array}{l}\text { T2: } 0.435 / 0.381 \\
\text { SRK/T: } 0.418 / 0.352 \\
\text { Holladay } 1: 0.455 / 0.389 \\
\text { T2 with H2.2 and optimized L: } 0.425 / 0.3648\end{array}$ \\
\hline
\end{tabular}

Only results concerning the studied formulas are shown. $P C I$ Partial coherence interferometry, OLCR Optical low coherence reflectometry 
method remains a relevant aim, even in the presence of new generation formulas. Additionally, a more accurate estimation of $\mathrm{H}$ might benefit eyes with steep or irregular corneas, such as those observed after refractive surgery or in the presence of keratoconus, where the use of a value closer to normal may lower prediction errors. The fact that the most important source of error in third generation formulas is the ACD estimation [16] makes the findings of this study relevant and points to ways for physicians to improve their calculations in highly myopic patients.

\section{Conclusions}

The T2 formula is recognized as the most precise option compared to the SRK/T and Holladay 1 formulas for the overall population (i.e. normal eyes). Nevertheless, evidence is contradictory regarding its accuracy in the highly myopic.

This paper provides evidence showing that T2 is less precise than SRK/T in the highly myopic eyes and describes a method to improve the corneal height estimation and the accuracy of the T2 formula.

A future study with more patients would be important in order to verify the findings in this paper. The addition of very long eyes, optimized constants, different intraocular lens designs and more formulas (like Olsen and Haigis) would allow for better comparison and confirmation of the effects found here.

\section{Appendix}

Steps for calculating corneal height using the SRK/T method:

1. Corneal radius of curvature, $r=337.5 / \mathrm{K}$.

2. Corrected axial length, LCOR:

If $\mathrm{L} \leq 24.2$ then $\mathrm{LCOR}=\mathrm{L}$

If $\mathrm{L} \geq 24.2$ then $\mathrm{LCOR}=-3.446+1.716 \mathrm{~L}-0.0237 \times \mathrm{L}^{2}$

3. Computed corneal width $\left(\mathrm{C}_{\mathrm{w}}\right)$

$$
\mathrm{C}_{\mathrm{w}}=-5.40948+0.58412 \times \mathrm{LCOR}+0.098 \times \mathrm{K}
$$

4. Corneal height $(\mathrm{H})$

$$
\begin{aligned}
& \mathrm{X}=\mathrm{r}^{2}-\left(\mathrm{Cw}^{2} / 4\right) \\
& \text { If } \mathrm{x}<0 \text { then } \mathrm{x}=0 \\
& \mathrm{H}=\mathrm{r}-\sqrt{\mathrm{X}}
\end{aligned}
$$

5. Offset for specific intraocular lens (IOL) to be implanted

$$
\text { Offset }=\mathrm{ACD}_{\text {const }}-3.336
$$

Steps in the T2 formula for calculating corneal height (H2).

$$
\mathrm{H} 2=-10.326+0.32630 \times \mathrm{L}+0.13533 \times \mathrm{K}
$$

Alternative formula for estimating T2 (H2.2).

$$
\mathrm{H} 2.2=-11.980+0.38626 \times \mathrm{LCOR}+0.14177 \times \mathrm{K}
$$

\section{Abbreviations}

95\% Cl: 95\% confidence interval; A: Constant used for SRK $T^{5}$; ACD: Anterior Chamber Depth; ACDconst: Constant used for anterior chamber depth in SRKT formula for specific IOL/surgeon; can be computed from A-constant ${ }^{5}$; Cw: Corneal width computed from L and $\mathrm{K}(\mathrm{mm})^{5}$; D: Diopters; ETDRS: Early Treatment Diabetic Retinopathy Study visual acuity test; $\mathrm{H}$ : Corneal Height theoretical estimation of the distance from a plane which lies above the anterior surface of the iris and the top of the central cornea at its endothelial surface, this model regards the cornea as a dome which base lies at the anterior iris. The corneal width and the corneal curvature are employed to estimate this value; $\mathrm{H} 2$ : Corneal Height Calculated with formula number 1 , described by Sheard et al. ; H2.2: Corneal Height Calculated with formula number 2, described by Sheard et al. ${ }^{7}$; HSRK T: Corneal Height Calculated with steps 2 to 4 of the SRK T formula ${ }^{5,7} ;$ IOL: Intraocular Lens; $\mathrm{K}$ : Keratometry. In Appendix 1 it refers exclusively to the averaged Keratometry where the abbreviation was kept in order to preserve the original description of the SRK $T^{5}$; L: Axial length measured using ultrasound in the original SRK $T$ T paper $^{5}$ and the IOL Master Biometer ${ }^{\oplus}(\mathrm{mm})$ in this paper; LCOR: Axial length with long eye correction; used in height formula ${ }^{5}$; LogMar: Logarithm of the Minimum Angle of Resolution; MAE: Mean Absolute Error; Max: Maximum; MedAE: Median Absolute Error;

Min: Minimum; mm: millimeters; $n$ : Number of eyes studied; offset: Difference between corneal height of the average eye and the ACD-constant of a given IOL ${ }^{5}$; OLCR: Optical low coherence reflectometry; PCl: Partial coherence interferometry; PostOp: Postoperative; PreOp: Preoperative; r: averaged corneal radius of curvature $(\mathrm{mm})^{5}$; SD: Standard Deviation; SN60W: Biconvex, Aspheric Intraocular lens model by Alcon ${ }^{\circledast}$, made of an Acrylate/Methacylate Copolymer; SRK/T: Third generation formula for intraocular lens calculation developed by Sanders, Retzlaff, and Kraff; T2: Formula developed by Sheard et al. for intraocular lens calculation based on the SRK $T^{7} ; \mathrm{T} 2.2$

OPTAL: Calculation of introaocular lens using two improvement methods for the SRK/T formula: the $\mathrm{H} 2.2$ formula for corneal height ${ }^{7}$ and the optimized axial length by Wang et al. ${ }^{13}$; VA: Visual acuity; $X$ : Mathematical estimation used as part of the calculation of the Corneal Height in the SRK/T formula; yo: years old; $\rho_{c}$ : Lin's concordance correlation coefficient

\section{Acknowledgements}

We would like to thank.

Dr. Edward Averbukh MD. Ophthalmologyst, Retina and Vitreous Specialist at Hadassah Ein Keren Hospital (Jerusalem, Israel), for his advice during the design of this study and providing the cases from the Hadassah Hospital sample group.

We sincerely thank Dr. Kenneth J. Hoffer, MD and Clinical Professor of Ophthalmology at UCLA, for his advice in the design of the present study. We want to acknowledge Dr. Richard M. Sheard, MD at the Royal Hallamshire Hospital, for providing the IOL calculation tool used in the present study.

We want to thank Clara López de Mesa, Epidemiologist at Clínica Barraquer, and the Research Department of Clínica Barraquer (Bogotá, Colombia) for the advice and aid regarding research methodology and statistics. Also Mireya Mora, Research Coordinator.

We would like to acknowledge Eduardo Fuentes, PhD and Ashley VanCott for aiding in the composition of this article.

Availability of data and material

The datasets generated and/or analysed during the current study are available in the Mendeley repository, https://data.mendeley.com/datasets/ nhgcnrjs9k/1, DOl: https://doi.org/10.17632/nhgenrjs9k.1

\section{Authors' contributions}

$\mathrm{Cl}$ : Contributed with the study design, collection, analysis and interpretation of the data and writing of the manuscript. GS: Contributed with the collection, interpretation of the data and writing of the manuscript. AMG: 
Contributed with the collection and interpretation of the data and writing of the manuscript. All authors have read and approved the manuscript.

\section{Authors' information}

Carlos Idrobo MD is an Ophthalmologyst and Retina and Vitreous specialist at the Eugenio Espejo Hospital, Quito - Ecuador. Former Retina and Vitreous Fellow in the Hadassah Ein Keren Hospital, Jerusalem - Israel and former Ophthalmology Resident in the Clínica Barraquer, Escuela Superior de Oftalmología - Instituto Barraquer de América, Bogotá Colombia. Gisella Santaella MD, is a Cataract and Refractive Surgery specialist in the Clínica Barraquer, Escuela Superior de Oftalmología - Instituto Barraquer de América, Cornea \& External Disease \& Refractive Surgery Fellow at the University of Toronto.

Ángela María Gutiérrez MD, is a Cornea, Cataract and Refractive Surgery specialist at the Clínica Barraquer, Dean of the Escuela Superior de Oftalmología - Instituto Barraquer de América and former President of The Sociedad Colombiana de Oftalmología.

\section{Funding}

No funding body participated in the present work. Authors were in charge of all costs related to study design, collection, analysis and interpretation of data as well as writing the manuscript.

\section{Ethics approval and consent to participate}

Ethics approval was sought and obtained from "Comité de Ética de la Clínica Barraquer", in Bogotá Colombia. All methods adhered to the Helsinki Declaration. The Institutional Review Board waived the need for written informed consent of the participants.

\section{Consent for publication}

Not applicable in this study.

\section{Competing interests}

The authors declare that they have no competing interests.

\section{Author details}

'Hospital Eugenio Espejo, Avenida Gran Colombia 170403, Quito, Ecuador. 2Escuela Superior de Oftalmología - Instituto Barraquer de América, Clínica Barraquer, Calle 100 N 18 A 51, Bogotá, Colombia.

Received: 7 March 2019 Accepted: 24 October 2019

Published online: 12 November 2019

\section{References}

1. Rajan MS, Keilhorn I, Bell JA. Partial coherence laser interferometry vs conventional ultrasound biometry in intraocular lens power calculations. Eye. 2002;16(5):552

2. Abulafia A, Barrett GD, Rotenberg $M$, et al. Intraocular lens power calculation for eyes with an axial length greater than $26.0 \mathrm{~mm}$ : comparison of formulas and methods. J Cataract Refract Surg. 2015:41(3):548-56.

3. Bang S, Edell E, Yu Q, Pratzer K, Stark W. Accuracy of intraocular Lens calculations using the IOLMaster in eyes with long axial length and a comparison of various formulas. Ophthalmology. 2011;118(3):503-6.

4. Retzlaff JA, Sanders DR, Kraff MC. Development of the SRKT intraocular lens implant power calculation formula. J Cataract Refract Surg. 1990; 16(3):333-40.

5. Haigis W. Occurrence of erroneous anterior chamber depth in the SRK/T formula. J Cataract Refract Surg. 1993;19(3):442-6.

6. Sheard RM, Smith GT, Cooke DL. Improving the prediction accuracy of the SRK/T formula: the T2 formula. J Cataract Refract Surg. 2010;36(11):1829-34.

7. Kane JX, Van Heerden A, Atik A, Petsoglou C. Intraocular lens power formula accuracy: comparison of 7 formulas. J Cataract Refract Surg. 2016;42(10): 1490-500.

8. Cooke DL, Cooke TL. Comparison of 9 intraocular lens power calculation formulas. J Cataract Refract Surg. 2016;42(8):1157-64

9. Optimized IOL constants for the ZEISS IOLMaster. http://ocusoft.de/ulib/c1. htm. Accessed 4 July 2016

10. Hoffer KJ, Aramberri J, Haigis W, et al. Protocols for Studies of Intraocular Lens Formula Accuracy. Am J Ophthalmol. 2015;160(3):403-405.e1.

11. Nickerson CAE. A Note On "A Concordance Correlation Coefficient to Evaluate Reproducibility". Biometrics. 1997;53(4):1503-7.
12. Wang L, Shirayama M, Ma XJ, Kohnen T, Koch DD. Optimizing intraocular lens power calculations in eyes with axial lengths above $25.0 \mathrm{~mm}$. J Cataract Refract Surg. 2011;37(11):2018-27.

13. Chong EW, Mehta JS. High myopia and cataract surgery. Curr Opin Ophthalmol. 2016;27(1):45-50.

14. Kim DH, Kim MK, Wee WR. Estimation of intraocular Lens power calculation after myopic corneal refractive surgery: using corneal height in anterior segment optical coherence tomography. Korean J Ophthalmol. 2015;29(3):195.

15. Kim S-Y, Cho SY, Yang JW, Kim CS, Lee YC. The correlation of differences in the ocular component values with the degree of myopic Anisometropia. Korean J Ophthalmol. 2013;27(1):44.

16. Jeong Jinho, Song Han, Lee Jimmy K, Chuck Roy S, Kwon Ji-Won, The effect of ocular biometric factors on the accuracy of various $1 \mathrm{OL}$ power calculation formulas, BMC Ophthalmology (2017) 17:62

\section{Publisher's Note}

Springer Nature remains neutral with regard to jurisdictional claims in published maps and institutional affiliations.

\section{Ready to submit your research? Choose BMC and benefit from:}

- fast, convenient online submission

- thorough peer review by experienced researchers in your field

- rapid publication on acceptance

- support for research data, including large and complex data types

- gold Open Access which fosters wider collaboration and increased citations

- maximum visibility for your research: over $100 \mathrm{M}$ website views per year

At $\mathrm{BMC}$, research is always in progress.

Learn more biomedcentral.com/submissions 